\title{
Discussion on the Reasons That Affect the Mental Health of Medical Postgraduate Students and Suggestions
}

\author{
Ya'nan Zheng ${ }^{1,2, *}$ Chunying Chang ${ }^{3}$
}

\author{
${ }^{1}$ Department of Psychology, Gannan Medical University, Ganzhou, Jiangxi, China \\ ${ }^{2}$ Rural Medical Education Research Center, Gannan Medical University, Ganzhou, Jiangxi, China \\ ${ }^{3}$ Office of Student Affairs, Jiangxi College of Applied Technology, Ganzhou, Jiangxi, China \\ ${ }^{* * *}$ Corresponding author. Email: marine873926@sina.com
}

\begin{abstract}
Students' mental health has been concerned by all sectors of society. However, at present, mental health education in colleges and universities is mainly targeted at college students, with few discussions at the postgraduate level. The mental health of medical postgraduate students needs more attention than that of other graduate students due to their professional features and the nature of their study. This paper analyzes the reasons that affect the mental health of medical postgraduate students from the perspectives of their studies, employment, characteristics of clinical medical work, doctor-patient relationship, scientific research pressure and lack of psychological intervention institutions, and puts forward specific suggestions in order to provide references for the reform and research of mental health education of medical postgraduate students.
\end{abstract}

Keywords: medical postgraduate, mental health, influencing factors, suggestion

\section{INTRODUCTION}

Influenced by the standardized training policies of resident physicians, the longer term of schooling, large number of courses, the larger pressure on employment and enrollment than other majors in college, the domestic doctor-patient relationship and the medical environment, the mental health level of medical students is more worthy of attention than that of other majors [1]. At present, a series of studies on the mental health of medical students have been carried out in China from multiple perspectives [2] [3] [4] [5] [6]. However, compared with medical undergraduates, academic circles pay less attention to the mental health of medical postgraduate students. With the reform of medical education and people's higher requirements for medical and health services, in recent years, medical and health institutions have increasingly strict requirements for medical graduates with master's degree. And there is a tendency for them to have higher requirements in their academic degree, scientific research ability and clinical skills. This also asks the training units to constantly raise their graduate requirements for masters of medicine. With the implementation of the "collaboration between the

*Fund project: Research Project on Degree and Postgraduate Education and Teaching Reform in Jiangxi Province in 2019 (JXYJG-2019-183). education and health sectors", under the new mode, and influenced by the "double examination" and "double identities", medical graduate have to face manifold pressure from scientific research, employment/studies, interpersonal relationship, marriage and family, theoretical study and clinical practice teaching, even the doctor-patient relationship and medical environment, etc. in three years. At the same time, with the dual identities as "students" and "medical staff" or "research assistants", medical postgraduate students face more psychological problems than medical undergraduates. Based on the above reasons, in the future, domestic medical colleges and universities should strengthen academic research on mental health of medical graduates and carry out targeted education and preventive measures. Combined with the practice of education and student management and literature arrangement, this paper analyzes the reasons affecting the mental health of medical graduates, and puts forward specific intervention plans, hoping to provide references for the development of graduate mental health education and student management in medical colleges. 


\section{REASONS}

\section{A. The impact of academic pressure}

Compared with the medical undergraduate stage, although there are fewer courses at the graduate stage, the time to complete the study is more limited. In general, medical postgraduate students should complete the theoretical knowledge study in the first year and participate in experiments in the laboratory or clinical medical work. However, the learning mode of medical postgraduate students is quite different from that of undergraduate students, which changes from "memorization" to "designability". At the same time, due to the short time to complete the study, most medical schools in the second year of graduate school need to complete the writing of academic papers, graduation thesis proposal, and begin their further research. In addition, medical postgraduate students in this stage also need to face the pressure of clinical practice. And many factors aggravate the negative effects of academic pressure and finally affect mental health.

\section{B. The impact of employment pressure}

The characteristics of medical and health work determine that practitioners need to be highly professional. At present, medical and health institutions continue to improve the qualifications of staff recruitment. The general recruitment requirements of third-grade class-A hospitals are doctoral students or excellent master's students, which have clinical skills, but also relatively high scientific research ability. In this context, it is difficult for them to enter the desired hospital after graduation. But there are a considerable number of graduate students who chose to take part in the postgraduate entrance exams because their employment unit was not ideal. Therefore, in face of the fact that it is still hard for them to realize their employment ideal and satisfy their expectations even with a master's degree, they naturally have psychology confusions which lead to psychological problems.

\section{The impact of clinical medical work}

Graduate medical students have received relatively systematic training in clinical medical practice at the undergraduate stage. However, in the postgraduate stage, their clinical learning is more professional, and they spend more time on practical learning in the hospital. As a result, their medical and health work is under greater pressure. Many tutors even assess and give work arrangement to the graduate students as department staff. At the same time, medical postgraduate students also need to complete experiments, do projects, and write papers. Under the great pressure of clinical treatment, they are more likely to fall into the contradiction between scientific research and clinical practice, leading to psychological problems.

\section{The impact of doctor-patient relationship and medical environment}

At present, the relationship between doctors and patients in China is complicated, and the problem of high pressure and high risk of medical personnel is increasingly serious. A large number of studies have shown that medical students' willingness to become doctors is declining [7] [8]. Compared with medical undergraduates, medical postgraduate students have a deeper understanding of doctor-patient relationship due to their long-term study in hospitals. They spend more time dealing with patients and their families and are more susceptible to negative doctor-patient relationship. In the face of the complex doctor-patient relationship, coping with the clinical medical work, scientific research, study, employment and admission to a higher grade, etc., medical master's students have decreased willingness to work as a doctor and suffer from career exhaustion, affecting their mental health.

\section{E. The impact of research pressure}

Medical postgraduate students have meet certain scientific research requirements for graduation, like publishing academic papers, accepting the tutor's scientific research training, doing experiments and completing the graduation thesis. Many colleges and universities make publication a basic requirement for graduation. Although the requirement for journals in which the papers are published is not high, faced with great clinical, academic and employment pressure, some medical postgraduate students will strive to publish high-quality papers in order to enter a better hospital. Yet scientific research also needs a lot of time, and they struggle to cope with it, which affects their mental health.

\section{F. The absence of mental health education and counseling institutions}

Mental health education in domestic colleges and universities is mainly aimed at college students, yet seldom carried out for graduate students. The reason may be that the teaching methods of graduate students are mostly small class, which is not suitable for largescale propaganda of mental health knowledge. And campus psychological activities, such as the "5.25 mental health education activity month" are also targeted at college students. However, after completing the theoretical study, most medical postgraduate students enter affiliated hospitals to learn clinical practice skills or enter laboratories for scientific research training, which makes it difficult to carry out mental health education on a large scale. And it is unrealistic to completely rely on the tutors to carry out mental health education for the graduate students. At 
the same time, there is a lack of service institutions similar to psychological counseling centers in universities in the hospital environment. Even when medical postgraduate students are aware of their psychological problems and seek counseling, there are few places to help them.

\section{SUGGESTIONS AND COUNTERMEASURES}

To sum up, the reasons affecting the mental health of medical postgraduate students are complicated. There are professional characteristics and personal factors, as well as the pressure of scientific research and other factors in the general environment of the training requirements for medical postgraduate students, which cannot only be attributed to the "lack of mental health education" and other reasons. Medical colleges and universities should take various aspects into consideration, provide effective mental health education for medical postgraduate students, carry out appropriate psychological intervention and publicity of mental health knowledge, and achieve the purpose of maintaining mental health.

\section{A. Offering mental health courses or integrating the content of mental health teaching into some courses}

At present, there is a lack of mental health education for medical postgraduate students. In view of this, medical colleges and universities can set up appropriate psychological health education courses for medical postgraduate students, and the teaching contents should be in line with the actual psychological problems of medical postgraduate students, such as emotional regulation, interpersonal communication technology, pressure regulation and career development. And the courses should not be set up in just the first semester, but in about four semesters. For example, in the first school year, the teaching contents can be pressure adjustment and adaptation to master learning, etc.; when it comes to the link of clinical practice, they can be interpersonal communication and emotional regulation, etc.; and when it comes to the completion of graduation thesis, they can be career planning and emotional regulation, etc. Teaching methods should not be limited to theoretical teaching. The methods of case analysis and student discussion, etc. which are suitable for the postgraduate stage should also be considered. For some medical colleges in which the teaching arrangement is relatively compact and it is difficult to set up mental health education course teaching alone, they can consider incorporating elements of mental health education into part of the curriculum. For example, interpersonal communication skills and other teaching contents can be included in humanistic medicine courses such as doctor-patient communication; professional courses closely related to clinical medicine, such as clinical psychology, can be considered; and adjustment means for scientific research pressure can be included in scientific research training, etc. It is necessary to improve the mental health of medical postgraduate students through targeted mental health education.

\section{B. Carrying out necessary mental health activities}

In view of the lack of necessary campus mental health activities for medical postgraduate students, it is viable to consider combining the characteristics of clinical medicine and scientific research to carry out necessary activities that can be integrated into the content of mental health, such as the investigation of depression in cancer patients and the common abnormal psychological analysis in each department of comprehensive hospitals and other research group discussion. Or clinical psychology experts can be invited to carry out lectures or scientific research design on common abnormal psychology, so as to promote medical postgraduates' attention to their own mental health by discussing abnormal psychology in academic or clinical practice.

\section{Strengthening the tutor's attention to the psychological problems so as to actively guiding the mental health of medical graduate students}

In the stage of postgraduate study, the influence of tutors on students is extremely important. Therefore, in the process of cultivating medical postgraduate students, it is necessary to give full play to the cultivation of students' mental health by tutors. First of all, regular training of mental health knowledge should be made to help them master the basic psychological communication skills and timely find the psychological problems of students. Secondly, in the process of clinical diagnosis and treatment, the tutor should introduce to the students the significance of mental health, especially the influence of healthy behavior and good mentality on the patients' rehabilitation, so as to help them to realize the value of mental health in life and medical treatment and thus to pay attention to their own psychological problems. Thirdly, the tutor should master the communication skills with students and the art of scientific research task assignment, so as to avoid the influence of excessive scientific research or clinical tasks on students' mentality. Finally, the tutor should communicate systematically with the students once a week to discuss the recent scientific research, clinical treatment and living conditions, so as to dispel students' doubts and maintain their mental health.

\section{Setting up postgraduate psychological counseling points in medical institutions according to the characteristics of medical institutions and graduate management}

In view of the relative lag in the development of psychological counseling work for medical 
postgraduate students, affiliated hospitals or other medical institutions that undertake the task of training medical postgraduate students can consider integrating mental health resources. For example, they can recruit graduate department administrators or counselors with psychological degrees as part-time psychological counselors and integrate the hospital psychological outpatient service and other institutions to carry out necessary psychological counseling for medical postgraduate students, timely solve the psychological problems of medical postgraduate students in need of psychological counseling and maintain mental health of students.

\section{E. Carrying out necessary interventions in a timely manner in view of the doctor-patient relationship and other issues much concerned by medical postgraduate students}

As for the situation of sharp doctor-patient relationship and the medical environment far below the expectation of medical postgraduate students, medical colleges and universities should carry out corresponding interventions. For example, they offer can humanistic medicine lectures, invite communication teachers to carry out small classes and clinical instruction teachers to instruct them on matters needing attention in communication. By helping them master communication skills and correctly understand the psychological state of patients, they can improve their understanding of doctor-patient relationship and reduce the impact of bad doctor-patient relationship on mental health. At the same time, it is necessary to strengthen the responsibility education of medical postgraduate students to help them to correctly understand the medical environment. Through the adjustment of cognition, they can maintain their inner balance, so as to adapt to medical and health work and improve the level of mental health.

\section{CONCLUSION}

The mental health education of medical postgraduates has been neglected by the mental health work in colleges and universities. Yet due to the influence of academic work, clinical work, scientific research, interpersonal relationship and other factors, their mental health is in urgent need of attention from medical colleges and universities. Only by developing targeted interventions according to the reasons that affect the mental health of medical graduate students, can the training goal of cultivating mentally and physically healthy senior medical and health professionals with both professional excellence and moral integrity be achieved.

\section{References}

[1] Sun Wenfang, Wang Changsheng, Zhao Ming, et al. Metaanalysis of Mental Health Status of Medical Students in China [J]. Modern Preventive Medicine, 2008, 45 (22): 4119-4125. (in Chinese)

[2] Wang Zhe, Li Hongjie, Wang Miao, et al. Influence of Past School Bullying Experience and Support on Anxiety State of Medical students [J]. Chinese Journal of School Health, 2019 40 (2): 236-238. (in Chinese)

[3] Zheng Yanan, Hu Wen. The Mediating Effect of Self-esteem Between Professional Psychological Help Attitude and Positive Psychological Capital of Students in a Medical College in Ganzhou City [J]. Medicine and Society, 2019, 32 (7): 99-102. (in Chinese)

[4] Zheng Yanan, Hu Wen. A Study on Positive Psychological Capital of Freshmen in a Medical University [J]. China Preventive Medicine, 2019, 20 (4): 276-279. (in Chinese)

[5] Gao Li, Zhang Qiumei, Zhang Dongfeng. Investigation on Stress Tolerance and Mental Health of Medical Students During Clinical Internship in a Medical College [J]. Chinese Journal of Health Education, 2019, 35 (10): 944-947. (in Chinese)

[6] Li Xuanxan, Zhang Xiumin, Wu Fangyuan, et al. Status of Anxiety, Depression and Its Relationship with Type A Behavior Among Medical Students in a Medical College in Changchun City [J]. Medicine and Society, 2008, 31 (2): 55-58, 62. (in Chinese)

[7] Guo Qian, Liu Huijun. The Investigation on Motivation and Professional Cognition of New Medical Students [J]. China Higher Medical Education, 2016 (1): 19-20. (in Chinese)

[8] Ruan Dengfeng, Ding Xiaoxia, Li Xiaobin, et al. Research on Medical Injury Incidents and Medical Students' Professional Attitudes [J]. Chinese medical ethics, 2016, 29 (2): 208-211. (in Chinese) 